\title{
SAÚDE ESCOLAR: RELAÇÃO ENTRE O SOBREPESO, OBESIDADE E SEDENTARISMO
}

\author{
Fabrícia de Freitas Faria Migliari
}

Universidade do Oeste Paulista - UNOESTE, Curso de Enfermagem, Presidente Prudente, SP. e-mail: fabriciamigliari@hotmail.com

\begin{abstract}
RESUMO
A escola é o cenário estratégico para a promoção de saúde através de atividades educativas e integrativas, identificando as necessidades e demandas dos adolescentes. $O$ objetivo desse trabalho foi Identificar a prevalência da obesidade e sua relação com o sedentarismo entre os adolescentes escolares, do ensino fundamental público de uma cidade do interior de São Paulo. Este trabalho é do tipo descritivo de abordagem quantitativa e com recorte único no tempo. A prevalência de sobrepeso neste estudo foi de $13,3 \%$ para sexo feminino e $23,4 \%$ para sexo masculino, para adolescentes classificados como obesos a prevalência foi de $9,6 \%$ para sexo feminino e $7,8 \%$ no sexo masculino. A presente pesquisa é de fundamental importância para a equipe de saúde das unidades básicas, pais, professores e escolares. Favorecendo o diagnóstico situacional para realizar ações preventivas e educativas, promovendo a saúde pública e melhor qualidade de vida para todos.
\end{abstract}

Palavras-chave: saúde escolar, atenção primária à saúde, promoção em saúde.

\section{SCHOOL HEALTH: RELATIONSHIP AMONG OVERWEIGHT, OBESITY AND SEDENTARY LIFESTYLE}

\section{ABSTRACT}

The school is the strategic setting for health promotion through educational and integrative activities, identifying the needs and demands of adolescents. The aim of this study was to identify the prevalence of obesity and its relationship to physical inactivity among schoolchildren, elementary school audience in a town in the interior of São Paulo. This work is descriptive quantitative approach and only cut in time. The prevalence of overweight in this study was $13.3 \%$ for females and $23.4 \%$ for male teenagers classified as obese, the prevalence was $9.6 \%$ for women and $7.8 \%$ in males. This research is of fundamental importance for the health team of the basic units, parents, teachers and school. Favoring the situational diagnosis to carry out preventive and educational activities, promoting public health and better quality of life for all.

Keywords: school health, primary health care, health promotion.

\section{INTRODUÇÃO}

A escola é o cenário estratégico para a promoção de saúde através de atividades educativas e integrativas, identificando as necessidades e demandas dos adolescentes ${ }^{1}$.

O profissional da saúde tem papel fundamental na promoção da saúde, na medida em que pode atuar em todos os seus componentes, realizando vários tipos de ações, tais como: promover atenção à saúde individual e ações de educação para a saúde na comunidade e estimular a participação efetiva da comunidade ${ }^{1}$.

A saúde escolar passou a ser considerada no Brasil no final do século XX, através da denominação Higiene Escolar, alcançada, sobretudo, do modelo de Política Médica alemã.
No final da década de 80 , através de um processo de reorganização, institui e adiciona no ambiente escolar o conceito de promoção da saúde, a fim de promover ações de planejamento e implementação de estratégias que identifique problemas de saúde na população infantojuvenil. Com o passar dos anos essas ações passam a constituir as diretrizes da nova política de atenção à saúde do escolar, e através do decreto no 6.286, de 5 de dezembro de 2007, cria-se o Programa Saúde na Escola (PSE) ${ }^{2}$.

O PSE é uma integração entre os Ministérios da Saúde e da Educação, visa oferecer atenção integral de prevenção, promoção e atenção à saúde de crianças, adolescentes e jovens, com vistas ao enfrentamento das vulnerabilidades que comprometem o pleno 
desenvolvimento de crianças e jovens da rede pública de ensino ${ }^{3}$.

É considerado adolescente o período dos 10 aos 19 anos, pois durante o seu crescimento e desenvolvimento são manifestas transformações anatômicas, fisiológicas, mentais e sociais ${ }^{4}$.

A fase da adolescência é marcada por grandes mudanças, no contexto físico, social e emocional, os mesmos passam a se preocupar com vários aspectos, como a identidade, os grupos ou "tribos" que irão se identificar, e principalmente na sua imagem corporal. Seu aspecto físico não é tão preocupante quanto seu peso, principalmente o sobrepeso ${ }^{5}$.

A alteração do peso pode estar relacionada com o sedentarismo que é a falta ou a prática insuficiente de atividade física. E atividade física é qualquer movimento corporal produzido pela musculatura esquelética, que ocasiona um gasto energético ${ }^{6}$.

Considera-se ativo 0 individuo que pratica atividade física moderada, pelo menos 20 min de atividade intensa em 3 ou mais dias da semana, ou $30 \mathrm{~min}$ de atividade moderada ou caminhada em 5 ou mais dias da semana ${ }^{7}$.

É conceituado muito ativo o individuo que pratica atividade física intensa por pelo menos 3 dias na semana, somando no mínimo $1.500 \mathrm{~min} / \mathrm{semana}$, ou a combinação de intensidade de atividade física, totalizando pelo menos $3.000 \mathrm{~min} / \mathrm{semana}^{7}$.

Nas ultimas décadas o sedentarismo tem se mostrado um fator importante para 0 desenvolvimento da obesidade em crianças e adolescentes de todos os níveis socioeconômicos, causando danos à saúde. Portanto, são muitas as evidências de que a prática regular de atividade física auxilia no controle e manutenção do peso corporal e traz benefícios para a saúde física, mental e social ${ }^{8-11}$.

Para realizar intervenções preventivas, é de fundamental importância à identificação dos determinantes da obesidade a fim de prevenir Doenças Crônicas Não Transmissíveis (DCNTs) e melhor qualidade de vida ao adolescente escolar.

Diante desse contexto surge uma questão: a obesidade entre os adolescentes está relacionada com o sedentarismo? Consequentemente surgem as seguintes hipóteses, uma provável prevalência de obesidade entre os adolescentes sedentários e obesidade pode está relacionada com 0 sedentarismo entre os adolescentes.
Neste sentido, o presente estudo tem como objetivo geral Identificar a prevalência da obesidade e sua relação com sedentarismo entre os adolescentes escolares, do ensino fundamental público de uma cidade do interior de São Paulo.

\section{MATERIAL E MÉTODOS}

Este estudo é do tipo descritivo de abordagem quantitativa e com recorte único no tempo. Está de acordo com a Resolução CNS 466/2012 e foi aprovado pelo Comitê de Ética em Pesquisa da Unoeste (CAAE: 11786612.0.0000.5515) para ser realizado a partir de dados coletados em um projeto de extensão universitária do Curso de Enfermagem da UNOESTE, que realiza promoção da saúde nas escolas. Para utilização dos dados houve autorização da coordenadora do Projeto de Extensão e do curso ao qual o Projeto de Extensão está vinculado. Os pais ou responsáveis assinaram termo de consentimento livre e esclarecido para participação dos adolescentes.

O projeto de extensão iniciou suas atividades em 2010, com a pretensão de colaborar na formação do profissional da saúde voltada para o SUS, desenvolvendo ação de prevenção à obesidade e promoção da qualidade de vida. As ações são desenvolvidas pelos estudantes do Curso de Enfermagem, como: as medidas antropométricas dos escolares e identificação das necessidades de saúde, sendo utilizado questionário auto aplicável pelo escolar e seu responsável. Os dados foram coletados pelos acadêmicos do Estágio Curricular Supervisionado, e ações de educação em saúde foram realizadas.

O Projeto foi realizado no Município de Presidente Prudente que possui 41 escolas do ensino médio, com aproximadamente $10 \mathrm{mil}$ matriculados ${ }^{12}$. Foram selecionadas intencionalmente três escolas do ensino médio, localizadas na região oeste do município, onde a docente responsável por esse trabalho realizava supervisão dos acadêmicos do Estagio Curricular Supervisionado do Curso de Enfermagem.

A população geral deste estudo foi de 316 alunos matriculados no $8^{\circ}$ ano, das três escolas públicas do ensino médio do município selecionadas. A população alvo foi constituída por apenas 147 alunos, os outros 169 alunos foram excluídos, devido a não autorização de alguns pais, outros não trouxeram a autorização 
dos pais, alguns faltaram no dia da coleta dos dados. Os Critérios de inclusão utilizados foram: alunos matriculados no $8^{\circ}$ ano, autorização dos responsáveis e preenchimento correto do questionário.

Os dados deste trabalho foram mensurados por meio dos instrumentos utilizados pelo Projeto de Extensão, entre fevereiro e março de 2013, constituídos por questionários de saúde respondido pelo próprio escolar e seu responsável em sua casa, e dados antropométricos mensurados na escola. Foram investigadas as seguintes variáveis: sexo e idade do adolescente, dados antropométricos, sinais vitais, condições de saúde, antecedentes familiares, padrão de sono e repouso, hábitos alimentares e de hidratação, recreação e lazer, atividades físicas, características sociais e emocionais. A análise dos dados foi realizada utilizando o programa Epi info Versão 3.5.2.

\section{RESULTADOS}

Fizeram parte deste estudo 147 adolescentes de três escolas públicas do interior de São Paulo, selecionados aleatoriamente alunos do $8^{\circ}$ ano do ensino fundamental, com faixa etária de 13 a 15 anos, sendo $64(43,5 \%)$ do sexo masculino e $83(56,5 \%)$ do sexo feminino.

A prevalência de sobrepeso neste estudo foi de $13,3 \%$ para sexo feminino e $23,4 \%$ para sexo masculino, para adolescentes classificados como obesos a prevalência foi de $9,6 \%$ para sexo feminino e $7,8 \%$ no sexo masculino.

No presente estudo podemos observar que $50 \%$ dos adolescentes classificados como sobrepeso e $61,5 \%$ dos adolescentes classificados como obesos são sedentários.

Constatou-se que, ocorreram variações entre a intensidade de atividade física realizada entre os sexos, no sexo feminino ocorreu prevalência de sedentarismo de $62,7 \%$ e de $53,2 \%$ para o sexo masculino, os adolescentes do sexo masculino são mais ativos do que os adolescentes do sexo feminino.

$\mathrm{Na}$ análise do tipo de atividade física realizada pelos adolescentes segundo sexo, encontrou-se diferenças entre os tipos de atividade física, sendo caminhada $70,9 \%$ para o sexo feminino e $23,3 \%$ para o sexo masculino, corrida $26,7 \%$ para o sexo masculino e $16,1 \%$ para sexo feminino e outras atividades sendo: futebol, vôlei e dança, foi de $40 \%$ para sexo masculino e $13 \%$ para sexo feminino.
Entre os escolares classificados como baixo peso o tipo de atividade física mais praticada foi a caminhada com $50 \%$, e os escolares classificados como obesos, o tipo de atividade física mais prevalente foi a caminhada com $80 \%$.

As categorias de atividades físicas observadas neste estudo em adolescentes classificadas como sobrepeso e obesidade podese destacar de maneira geral, a caminhada (80\%), em seguida da corrida $(38,4 \%)$.

\section{DISCUSSÃO}

Os resultados desse estudo puderam caracterizar o perfil das condições de saúde dos adolescentes escolares, corroborando com outras pesquisas realizadas no país. Devemos consideram as peculiaridades de cada região e comunidade, para que intervenções mais assertivas sejam realizadas de acordo com a realidade vivenciada.

Em outras regiões do Brasil há variações nos valores dos resultados encontrados em relação à prevalência de sobrepeso entre adolescentes, entretanto, sempre se encontram entre a faixa de $13 \%$ a $27 \%$. O sobrepeso na adolescência poderá ocasionar obesidade na fase adulta, ocorrendo agravos à saúde. Portanto, fazse necessário a identificação precoce de sobrepeso em adolescentes, na busca da prevenção de morbimortalidades relacionadas ${ }^{13}$.

Considerando o risco de morbidade por coronariopatia e aterosclerose que vem aumentando entre homens e mulheres sobrepesos ainda na adolescência ${ }^{13}$, torna-se importante avaliação clínica e ações preventivas nessa população.

Em estudo realizado em escolas do Município de Capão da Canoa, Rio Grande do Sul, $35 \%$ dos escolares sobrepesos e obesos eram sedentários ${ }^{11}$, corroborando com os resultados desse estudo.

O resultado desse estudo contribuiu para o planejamento de novas ações de promoção de saúde entre escolares. Há 3 anos os estudantes de Enfermagem da UNOESTE, Presidente Prudente/SP realizam educação em saúde em escolas do município, tendo como tema principal a prevenção de obesidade. Essa ação é realizada durante práticas e estágios nas unidades da Atenção Básica de forma continuada, por meio de oficinas, grupos, orientações e acompanhamento clínico da comunidade escolar. 
A partir dos resultados dessa pesquisa, conclui-se que a prevalência de sobrepeso e obesidade entre adolescentes escolares é significativa. Entretanto, o sedentarismo não teve relação com o IMC, os adolescentes com excesso de peso corporal não apresentam maior prevalência de sedentarismo e adolescentes com peso adequado nem sempre praticam atividades físicas.

\section{CONFLITO DE INTERESSE}

Os autores declaram não haver qualquer potencial de conflito de interesse que possa interferir na imparcialidade deste trabalho científico.

\section{REFERÊNCIAS}

1. Maciel ELN, Oliveira CB, Frechiani JM, Sales CMM, Brotto, LDA, Araujo MD. Projeto Aprendendo Saúde na Escola: a experiência de repercussões positivas na qualidade de vida e determinantes da saúde de membros de uma comunidade escolar em Vitória, Espírito Santo. Ciên Saúde Col. 2010;15(2):389-96.

2. Lacerda BL. Evolução da saúde do escolar no Brasil e a inserção do enfermeiro: uma revisão bibliográfica. Acesso em: 08 mai 15. Disponível em: http://www.webartigos.com

3. Brasil. Ministério da Saúde. Secretaria de Atenção à Saúde. Departamento de Atenção Básica. Orientações para a coleta e análise de dados antropométricos em serviços de saúde: Norma Técnica do Sistema de Vigilância Alimentar e Nutricional - SISVAN. Brasília, 2011. Acesso em: 11 mai 15. Disponível em: http://189.28.128.100/nutricao/docs/geral/orientacoe $\mathrm{s}$ coleta analise dados antropometricos.pdf

4. Rocha RCL, Souza E, guazzelli CAF, chambô Filho A, Soares EP, Nogueira ES. Prematuridade e baixo peso entre recém-nascidos de adolescentes primíparas. Rev Bras Ginecol Obstet. 2006;28(9):530-5.

5. Fernandes FM. Alimentação e nutrição entre escolares: caso dos alunos de uma escola do município, Vitória, ES. 2006. 49f. [Monografia]. Curso de Pós-Graduação Especialização em Nutrição Clínica, Universidade Veiga de Almeida, Vitória, 2006.

6. Cunha IC, Peixoto MRG, Jardim PCBJ, Alexandre VP. Fatores associados à prática de atividade física na população adulta de goiânia: monitoramento por meio de entrevistas telefônicas. Rev Bras Epidemiol. 2008;11(3):495-504.

DOI:

http://dx.doi.org/10.1590/S1415-

790X2008000300016

7. Ferreira SS. Prevalência de sedentarismo e fatores associados em adolescentes da região Leste da cidade de Goiânia, GO, 2011. 77f. [Dissertação]. Mestrado em Ciências da Saúde. Programa de Pôs- Graduação em
Ciências da Saúde da Universidade Federal de Goiás, 2011.

8. Baruki SBS, Rosado LEFPL, Rosado GP, Ribeiro RCL. Associação entre estado nutricional e atividade física em escolares da Rede Municipal de Ensino em Corumbá, MS, Rev Bras Med Esporte. 2006;12(2):90-4. DOI: http://dx.doi.org/10.1590/S1517$\underline{86922006000200007}$

9. Bergmann GG, Araújo MLB, Garlipp DC, Lorenzi TDC, Gaya A. Alteração anual no crescimento e na aptidão física relacionada á saúde de escolares. Rev Bras Cineantropom Desempenho Hum. 2005;7:55-61.

10. Jenovesi JF, Bracco MM, Colugnati FAB, Taddei JAAC. Perfil de atividade física em escolares da rede pública de diferentes estados nutricionais. Rev Bras Ciên Mov. 2003;11(4):57-62.

11. Silva KS, Nahas MV, Hoefelmann LP, Lopes AS, Oliveira ES. Associações entre atividade física, índice de massa corporal e comportamentos sedentários em adolescentes. Rev Bras Epidemiol. 2008;11(1):159-68. DOI: http://dx.doi.org/10.1590/S1415-

\section{X2008000100015}

12. IBGE - Instituto Brasileiro de Geografia e Estatística. Antropometria e estado nutricional de crianças, adolescentes e adultos no Brasil. Rio de Janeiro, RJ, Brasil, 2010. Acesso em: 09 mai 15. Disponível em: http://www.ibge.gov.br/home/estatistica/populacao/ condicaodevida/pof/2008 2009 encaa/

13. Silva RCR, Malina RM. Nível de atividade física em adolescentes do Município de Niterói, RJ, Brasil. Cad Saúde Públ. 2000;16(4):1091-7.

Recebido para publicação em 28/08/2015

Revisado em 31/08/2015

Aceito em 04/09/2015 